\title{
THE WEAK COTYPE 2 AND THE ORLICZ PROPERTY OF THE LORENTZ SEQUENCE SPACE $d(a, 1)$
}

\author{
by J. ZHU
}

(Received 15 March, 1991)

1. Preliminaries. The question "Does a Banach space with a symmetric basis and weak cotype 2 (or Orlicz) property have cotype 2 ?" is being seriously considered but is still open though the similar question for the r.i. function space on $[0,1]$ has an affirmative answer. (If $X$ is a r.i. function space on $[0,1]$ and has weak cotype 2 (or Orlicz) property then it must have cotype 2 .) In this note we prove that for Lorentz sequence spaces $d(a, 1)$ they both hold.

Let $a_{1} \geq \ldots \geq a_{n} \geq \ldots>0$. The Lorentz sequence space $d(a, 1)$ is the space of vectors in $\mathbf{R}^{\infty}$ with finite norm given by

$$
\|x\|=\sum_{i} a_{i} x_{i}^{*}
$$

where $\left(x_{i}^{*}\right)$ is the decreasing rearrangement of $\left(\left|x_{i}\right|\right)$.

A decreasing positive sequence $a=\left(a_{n}\right)$ is said to be $p$-regular $(p \geq 0)$ if there exists a constant $0<C<\infty$ such that

$$
\sum_{i \leq n} a_{i}^{p} \leq C n a_{n}^{p} \quad(n \in \mathbf{N})
$$

Reisner proved in [4] that $a$ is 1-regular if and only if $d(a, 1)$ does not contain $l_{\infty}^{n}$ uniformly, which is also equivalent to the existence of $q<\infty$ such that $d(a, 1)$ is $q$-concave (for definition of $q$-concave see below). Another result in [4] which plays an important role in our proof is that $d(a, 1)$ is 2-concave if and only if $a$ is 2-regular. It is well known that for Banach lattices 2-concave and cotype 2 are equivalent. See [2].

The Banach space $X$ is of weak cotype 2 if there is a constant $c \geq 0$, such that for any $n \in \mathbf{N}$ and $n$-dimensional subspace $E \subset X$, we have $\delta(E) \geq c n^{1 / 2}$ where

$$
\begin{aligned}
& \delta(E):=\sup \left\{\rho_{2}\left(x_{1}, \ldots, x_{k}\right)\right. \\
&\left.=\left(\frac{1}{2^{k}} \sum_{\epsilon_{i}= \pm 1}\left\|\sum_{i \leq k} \epsilon_{i} x_{i}\right\|^{2}\right)^{1 / 2}: x_{1}, \ldots, x_{k} \in E, \mu_{2}\left(x_{1}, \ldots, x_{k}\right) \leq 1, k \in \dot{N}\right\} \\
& \mu_{p}\left(x_{1}, \ldots, x_{k}\right):=\sup \left\{\left(\sum_{i \leq k}\left|f\left(x_{i}\right)\right|^{p}\right)^{1 / p}: f \in E^{*},\|f\| \leq 1\right\} \quad(1 \leq p \leq \infty) .
\end{aligned}
$$

It is well known that weak cotype 2 implies cotype $q$ for any $q>2$. See [3] for details of weak cotype 2 .

The Banach space $X$ has the Orlicz property if there exists a constant $C$ such that for any $k \in \mathbf{N}$ and any $x_{1}, \ldots, x_{k} \in X$, we have

$$
\left(\sum_{i \leq k}\left\|x_{i}\right\|^{2}\right)^{1 / 2} \leq C \mu_{1}\left(x_{1}, \ldots, x_{k}\right) .
$$

The Orlicz constant $\pi_{2,1}(X):=\inf C$. For more information about the Orlicz property see [1].

Glasgow Math. J. 34 (1992) 271-276. 
A Banach lattice $X$ is said to be $q$-concave $(q \geq 2)$ if there exists a constant $D$ such that

$$
\left(\sum_{i \leq k}\left\|x_{i}\right\|^{q}\right)^{1 / q} \leq D\left\|\left(\sum_{i \leq k}\left|x_{i}\right|^{q}\right)^{1 / q}\right\|
$$

for any $k \in \mathbf{N}$ and any $x_{1}, \ldots, x_{k}$ in $X$.

\section{The weak cotype 2}

Theоrem 1.d(a,1) is of weak cotype 2 if and only if it is of cotype 2.

Proof. Assume $d(a, 1)$ is of weak cotype 2 , so $d(a, 1)$ does not contain $l_{\infty}^{n}$ uniformly. By [4] $d(a, 1)$ is $q$-concave for some $q<\infty$ and $a$ is 1-regular. For any $n \in \mathbf{N}$, let $d(a, 1 ; n)$ denote the $n$-dimensional subspace of $d(a, 1)$ with all coordinates after the $n$th being 0 . Then the $q$-concave constant of $d(a, 1 ; n)$ is independent of $n$.

Let $x_{1}, \ldots, x_{k} \in d(a, 1 ; n)$. Then we have, using Hölder's inequality, the definition of $q$-concavity and Khintchine's inequality,

$$
\begin{aligned}
\rho_{2}\left(x_{1}, \ldots, x_{k}\right) & \leq\left(\frac{1}{2^{k}} \sum_{\epsilon_{i}= \pm 1}\left\|\sum_{i \leq k} \epsilon_{i} x_{i}\right\|^{q}\right)^{1 / q} \\
& \leq C_{1}\left\|\left(\frac{1}{2^{k}} \sum_{\epsilon_{i}= \pm 1}\left|\sum_{i \leq k} \epsilon_{i} x_{i}\right|^{q}\right)^{1 / q}\right\| \\
& \leq C_{2}\left\|\left\{\left(\sum_{i \leq k} x_{i}^{2}(j)\right)^{1 / 2}\right\}_{j=1}^{n}\right\| .
\end{aligned}
$$

Without loss of generality we may assume that the numbers $\left(\sum_{i \leq k} x_{i}^{2}(j)\right)^{1 / 2}$, for $j=1, \ldots, n$, are decreasing. Then from (1) one has

$$
\rho_{2}\left(x_{1}, \ldots, x_{k}\right) \leq C_{2} \sum_{j \leqslant n}\left(\sum_{i \leq k}\left|x_{i}(j)\right|^{2}\right)^{1 / 2} a_{j}
$$

Suppose that $\mu_{2}\left(x_{1}, \ldots, x_{k}\right)=1$. Since $y_{\epsilon}=\left\{\epsilon_{j} a_{j}\right\}_{j=1}^{n} \in d(a, 1 ; n)^{*}$ has norm 1 for any $\epsilon=\left(\epsilon_{1}, \ldots, \epsilon_{n}\right) \in\{-1,1\}^{n}$, one has

$$
\sum_{i \leq k}\left(y_{\epsilon}, x_{i}\right)^{2} \leq 1
$$

Averaging over $\{-1,1\}^{n}$ we have

$$
\sum_{j \leq n}\left(\sum_{j \leq k}\left|x_{i}(j)\right|^{2}\right) a_{j}^{2} \leq 1
$$

Combining (2), (3) and Lemma 1 below we have

$$
\delta(d(a, 1 ; n)) \leq C_{3} \frac{\sum_{j \leq n} a_{j}}{\left(\sum_{j \leq n} a_{j}^{2}\right)^{1 / 2}}
$$


Meanwhile $\delta(d(a, 1 ; n)) \geq c n^{1 / 2}$, so that

$$
\left(n \sum_{j \leq n} a_{j}^{2}\right)^{1 / 2} \leq C_{4} \sum_{j \leq n} a_{j},
$$

for $\forall n \in \mathbf{N}$. Hence $a$ is 2-regular, since $a$ is 1-regular, and so $d(a, 1)$ is 2-concave by [4], equivalently of cotype 2 .

LeMmA 1. Let $a=\left(a_{1}, \ldots, a_{n}\right), \quad x=\left(x_{1}, \ldots, x_{n}\right)$, with $a_{1} \geq \ldots \geq a_{n} \geq 0$ and $x_{1} \geq \ldots \geq x_{n} \geq 0$. Write ax $=\left(a_{1} x_{1}, \ldots, a_{n} x_{n}\right)$. Then

$$
\frac{\|a x\|_{1}}{\|a x\|_{2}} \leq \frac{\|a\|_{1}}{\|a\|_{2}} .
$$

Proof. Define $R\left(x_{1}, \ldots, x_{n}\right)=\|a x\|_{1} /\|a x\|_{2}$ for any $x$ as above. We show that for $1 \leq k \leq n-1$, we have

$$
R\left(x_{1}, \ldots, x_{k}, x_{k}, \ldots, x_{k}\right) \geq R\left(x_{1}, \ldots, x_{k}, x_{k+1}, \ldots, x_{k+1}\right) .
$$

Then the conclusion follows because $R\left(x_{1}, \ldots, x_{1}\right)=\|a\|_{1} /\|a\|_{2}$. Define

where

$$
F(t)=R\left(x_{1}, \ldots, x_{k}, t, \ldots, t\right)=\frac{A+B t}{\sqrt{C+D t^{2}}},
$$

Then

$$
\begin{array}{ll}
A=\sum_{i \leq k} a_{i} x_{i}, & B=\sum_{j=k+1}^{n} a_{j}, \\
C=\sum_{i \leq k} a_{i}^{2} x_{i}^{2}, & D=\sum_{j=k+1}^{n} a_{j}^{2} .
\end{array}
$$

$$
\begin{aligned}
F^{\prime}(t) & =\left[B\left(C+D t^{2}\right)-(A+B t) D t\right]\left(C+D t^{2}\right)^{-3 / 2} \\
& =(B C-A D t)\left(C+D t^{2}\right)^{-3 / 2} .
\end{aligned}
$$

If $0 \leq t \leq x_{k}$ (and hence $t \leq x_{i}$, for $\left.i \leq k\right)$, then

$$
A D t=\sum_{1 \leq i \leq k} \sum_{k+1 \leq j \leq n} a_{i} a_{j}^{2} t x_{i} \leq \sum_{i} \sum_{j} a_{i}^{2} a_{j} x_{i}^{2}=B C .
$$

Hence $F^{\prime}(t) \geq 0$, and so $F(t)$ is increasing. The statement follows.

\section{Some results on Lorentz sequence spaces.}

Lemma 2. (1) $\mu_{2}\left(e_{1}, \ldots, e_{n}\right)=\sqrt{\sum_{i \leq n} a_{i}^{2}}$ in $d(a, 1)$.

(2) $\pi_{2,1}(d(a, 1)) \geq \sqrt{n} \frac{a_{1}}{\sum_{i \leq n} a_{i}}$ for each $n$.

(3) If $a$ is 1-regular, then for $k \geq l$,

$$
l\left(a_{k+1}^{2}+\ldots+a_{k+1}^{2}\right) \leq 4 C^{2}\left(a_{k+1}+\ldots+a_{k+1}\right)^{2},
$$

where $C$ is a constant independent of $k$ and $l$. 
Proof. (1) and (2) are fairly easy. We only prove (3). By 1-regularity of $a$, there is a constant $C$ such that

$$
(k+1) a_{k+1} \leq a_{1}+\ldots+a_{k+l} \leq(k+l) C a_{k+l}
$$

and so $a_{k+1} \leq \frac{k+l}{k+1} C a_{k+l} \leq 2 C a_{k+l}$. Hence

$$
l\left(a_{k+1}^{2}+\ldots+a_{k+l}^{2}\right) \leq l^{2} a_{k+1}^{2} \leq 4 C^{2} l^{2} a_{k+1}^{2} \leq 4 C^{2}\left(a_{k+1}+\ldots+a_{k+l}\right)^{2},
$$

which completes the proof.

We now prove a result using Talagrand's "isoperimetric inequality" (see [6] for details). Let $x_{1}, \ldots, x_{n}$ be elements of any linear normed space $X, \mu_{2}\left(x_{1}, \ldots, x_{n}\right)=\sigma$. Let $P_{n}$ be the usual probability measure on $D_{n}=\{-1,1\}^{n}$. For $\epsilon \in D_{n}$, write $s(\epsilon)=\sum_{i} \epsilon_{i} x_{i}$. For $t>0$, let $A_{t}=\left\{\epsilon \in D_{n}:\|s(\epsilon)\| \leq t\right\}, B_{t}=D_{n} \backslash A_{t}$. The inequality states that for $M>0$, $P_{n}\left(A_{M}\right) P_{n}\left(B_{M+1}\right) \leq \exp \left(-t^{2} / 8 \sigma^{2}\right)$. If $M=\int\|s(\epsilon)\|$, then $P_{n}\left(B_{2 M}\right) \leq \frac{1}{2}$ by since Chebyshev's inequality $M \geq \int_{B_{2 M}}\|s(\epsilon)\| \geq 2 M P_{n}\left(B_{2 M}\right)$. Hence $P_{n}\left(B_{2 M+t}\right) \leq 2 \exp \left(-t^{2} / 8 \sigma^{2}\right)$.

Proposition. Let $y_{1}, \ldots, y_{N}$ be positive elements of $d(a, 1 ; n)$ with $\sum_{i \leq N} y_{i}^{2} \leq 1$. Let the $q$-concave constant of $d(a, 1 ; n)$ be $C$, for some $q<\infty$. Then there exist $z_{1}, \ldots, z_{N} \in$ $d(a, 1 ; n)$ and $C^{\prime}=C^{\prime}(C)$ such that $\left|z_{i}(j)\right|=y_{i}(j)(\forall i, j)$ and

$$
\mu_{1}\left(z_{1}, \ldots, z_{N}\right) \leq C^{\prime}\left(\sum a_{i}+\sqrt{N \sum a_{i}^{2}}\right)
$$

This generalizes Lemma 6.4 of [5]. The proof is similar but more transparent.

Proof. Let $D_{N n}$ denote the set of $\epsilon=\left\{\epsilon_{i, j}: i \leq N, j \leq n\right\}$ with $\epsilon_{i, j} \in\{-1,1\}$. Assuming equal probability $2^{-N n}$ to each such $\epsilon$, define $z_{i}^{\epsilon}(j)=\epsilon_{i, j} y_{i}(j)$ for $1 \leq i \leq N, 1 \leq j \leq n$. Clearly $\left|z_{i}^{\epsilon}(j)\right|=y_{i}(j)$.

Let $\eta_{i} \in\{-1,1\}$ for $1 \leq i \leq N$. Then

$$
\sum_{i} \eta_{i} z_{i}^{\epsilon}(j)=\sum_{i} \epsilon_{i, j}^{*} y_{i}(j)=\sum_{i} z_{i}^{\epsilon^{*}}(j)
$$

where $\epsilon_{i, j}^{*}=\eta_{i} \epsilon_{i, j}$. The map $\epsilon \mapsto \epsilon^{*}$ maps $D_{N n}$ onto itself.

Write $y_{i, j}=y_{i}(j) e_{j}$, so that

$$
z_{i}^{\epsilon}=\sum_{i} \epsilon_{i, j} y_{i, j}
$$

If $f$ is a linear functional on $d(a, 1 ; n)$ with $\|f\| \leq 1$, then

$$
\begin{aligned}
\sum_{i} \sum_{j} f\left(y_{i, j}\right)^{2} & =\sum_{j} \sum_{i} y_{i}(j)^{2} f\left(e_{j}\right)^{2} \\
& \leq \sum_{j} f\left(e_{i}\right)^{2} \leq \sum_{i} a_{i}^{2}
\end{aligned}
$$

by (1) of Lemma 2 and $\sum_{i} y_{i}(j)^{2} \leq 1$. Hence $\mu_{2}\left\{y_{i, j}: i \leq N, j \leq n\right\} \leq \sqrt{k a_{i}^{2}}$. 
We will show that

$$
\int_{D_{N n}}\left\|\sum_{i, j} \epsilon_{i, j} y_{i, j}\right\| d P(\epsilon) \leq C^{\prime} \sum a_{i} .
$$

By the isoperimetric inequality it will then follow that

$$
P_{N n}\left\{\epsilon:\left\|\sum_{i} z_{i}^{\epsilon}\right\| \geq C^{\prime} \sum a_{i}+t\right\} \leq 2 \exp \left(-t^{2} / 8 \sum a_{i}^{2}\right) .
$$

If we then choose $t$ with $t^{2}=8 N \sum a_{i}^{2}$, then for each $\left(\eta_{i}\right) \in D_{N}$, we have

$$
P_{N n}\left\{\epsilon:\left\|\sum \eta_{i} z_{i}^{\epsilon}\right\| \geq C^{\prime} \sum a_{i}+t\right\} \leq 2 e^{-N} \text {. }
$$

Hence the probability of the union of such sets for all $\left(\eta_{i}\right)$ is not greater than $2^{N+1} e^{-N}(<1$, if $N>3)$. So there is an $\epsilon$ belonging to none of these sets, so that $\left\|\sum \eta_{i} z_{i}^{\epsilon}\right\| \leq C^{\prime} \sum a_{i}+t$ for all $\left(\eta_{i}\right)$. To prove (5), note first that for each $s, \sum_{j} y_{i, j}(s)^{2}=$ $y_{i}(s)^{2}$, and so $\sum_{i, j} y_{i, j}(s)^{2}=\sum_{i} y_{i}(s)^{2} \leq 1$. By the $q$-concavity and Khintchine's inequality, it is easy to obtain the statement.

\section{The Orlicz property.}

TheOREM 2. If $d(a, 1)$ has the Orlicz property then $a$ is 2-regular; equivalently $d(a, 1)$ is of cotype 2 .

Proof. Throughout the proof we use $M, M_{1}, M_{2}, \ldots$ for the constants which are independent of $n$. Since $\pi_{2,1}(d(a, 1))<\infty, d(a, 1)$ does not contain $l_{\infty}^{n}$ uniformly, so by [4], $a$ is 1-regular. Then it is enough to prove that there exists $M$ such that $n \sum_{i \leq n} a^{2} \leq$ $M\left(\sum_{i \leq n} a_{i}\right)^{2}, \forall n \in \mathbf{N}$.

Without loss of generality we may assume that $n=2^{m}$. Let

$$
k^{\prime}=\frac{\left(\sum_{i \leq n} a_{i}\right)^{2}}{\sum_{i \leq n} a_{i}^{2}} .
$$

Then $1 \leq k^{\prime} \leq n$. Let $k$ by the largest integer of the form $2^{s}$ with $k \leq k^{\prime}$, so that $2 k \geq k^{\prime}$. Note that

$$
\left(k \sum_{i \leq n} a_{i}^{2}\right)^{1 / 2} \leq \sum_{i \leq n} a_{i} \leq\left(2 \sum_{i \leq n} a_{i}^{2}\right)^{1 / 2} .
$$

Let $l=n / k$ (an integer). We must show that $l \leq M_{1}$. Let

$$
y(m)=a_{(m-1) l+1}+\ldots+a_{m l}, \quad m=1, \ldots, k,
$$

so that $y(1) \geq \ldots \geq y(k)$. Let $y_{1}=y \in \mathbf{R}^{k}$ and let $y_{2}, \ldots, y_{k}$ be the elements of $\mathbf{R}^{k}$ 
obtained from $y$ by cyclic permutation. Let $x_{i}$ be the element $\left(y_{i}, \ldots, y_{i}\right)$ of $\left(\mathbf{R}^{k}\right)^{l}=\mathbf{R}^{n}$ for $1 \leq i \leq k$. Then

$$
\begin{aligned}
\left\|x_{i}\right\|= & y(1)\left(a_{1}+\ldots+a_{l}\right)+y(2)\left(a_{l+1}+\ldots+a_{2 l}\right) \\
& +\ldots+y(k)\left[a_{(k-1) l+1}+\ldots+a_{k l}\right]=\sum_{j \leq k} y(j)^{2}=\|y\|_{2}^{2} .
\end{aligned}
$$

Also $\left(\sum_{i \leq k} x_{i}^{2}\right)^{1 / 2}=\|y\|_{2}(1, \ldots, 1)$.

Since $d(a, 1)$ is $q$-concave for some $q<\infty$, by the Proposition there exist $z_{1}, \ldots, z_{k}$ such that $\left|z_{i}\right|=x_{i}$ (hence $\left\|z_{i}\right\|=\|y\|_{2}^{2}$ ) and

$$
\mu_{1}\left(z_{1}, \ldots, z_{k}\right) \leq\|y\|_{2} M_{2} \sum_{i \leq n} a_{i} .
$$

Since $\left(\sum\left\|z_{i}\right\|^{2}\right)^{1 / 2} \leq \pi_{2,1}(d(a, 1)) \mu_{1}\left(z_{1}, \ldots, z_{k}\right)$, we have

$$
\|y\|_{2} \leq M_{3}\left(\sum_{i \leq n} a_{i}^{2}\right)^{1 / 2} \text {. }
$$

By (2) of Lemma $2, \sqrt{l}\left(a_{1}^{2}+\ldots+a_{l}^{2}\right) \leq M_{4} y(1)^{2}$, and by (3) of Lemma 2

$$
l\left(a_{(m-1) l+1}^{2}+\ldots+a_{m l}^{2}\right) \leq M_{5} y(m)^{2} \quad(m=2, \ldots, k) .
$$

Hence

$$
\sqrt{l} \sum_{i \leq n} a_{i}^{2} \leq M_{6}\|y\|_{2}^{2} \leq M_{7} \sum_{i \leq n} a_{i}^{2}
$$

and so $l \leq M_{1}$.

ACKNowledgement. The author wishes to thank Dr. Jameson for his generous help in rewriting the original version and many valuable discussions.

\section{REFERENCES}

1. G. J. O. Jameson, Summing and nuclear norms in Banach space theory (Cambridge University Press, 1987).

2. J. Lindenstrauss and L. Tzafriri, Classical Banach spaces II (Springer-Verlag, 1977).

3. G. Pisier, The volume of convex bodies and Banach space geometry (Cambridge University Press, 1989).

4. S. Reisner, A factorization theorem in Banach lattices and its application to Lorentz spaces, Ann. Inst. Fourier 31 (1981), 239-255.

5. M. Talagrand, An isoperimetric inequality on the cube and the Kintchine-Kahane inequality, Proc. Amer. Math. Soc. 104 (1989), 905-909.

6. M. Talagrand, Cotype of operators from $C(K)$, to appear.

\section{Department of Mathematics}

UNIVERSITY OF LANCASTER

LANCASTER LA1 4YF

ENGLAND 